\title{
Vision-based adaptive variable rate spraying approach for unmanned aerial vehicles
}

\author{
Linhui Wang ${ }^{1,2}$, Yubin Lan ${ }^{1,3}$, Xuejun Yue ${ }^{1,2^{*}}$, Kangjie Ling ${ }^{1,2}$, Zhenzhao Cen ${ }^{1,2}$, \\ Ziyao Cheng ${ }^{1,2}$, Yongxin Liu ${ }^{1,4}$, Jian Wang ${ }^{1,2}$ \\ (1. International Laboratory of Agricultural Aviation Pesticide Spraying Technology (ILAAPST), Guangzhou 510642, China; \\ 2. College of Electronic Engineering, South China Agricultural University, Guangzhou 510642, China; \\ 3. College of Engineering, South China Agricultural University, Guangzhou 510642, China; \\ 4. School of Civil Engineering and Transportation, South China University of Technology, Guangzhou 510640, China)
}

\begin{abstract}
The rapid developments of unmanned aerial vehicles (UAV) and vision sensor are contributing a great reformation in precision agriculture. Farmers can fly their UAV spraying pesticides around their crop fields while staying at their remote control room or any place that is separated from their farm land. However, there is a common phenomenon in rice planting management stage that some empty areas are randomly located in farmland. Therefore, a critical problem is that the waste of pesticides that occurs when spraying pesticides over rice fields with empty areas by using the common UAV, because it is difficult to control the flow accuracy based on the empty areas changing. To tackle this problem, a novel vision-based spraying system was proposed that can identify empty areas automatically while spraying a precise amount of pesticides on the target regions. By this approach, the image was preprocessed with the Lucy-Richardson algorithm, then the target area was split from the background with $k$-means and the feature parameters were extracted, finally the feature parameters were filtered out with a positive contribution which would serve as the input parameters of the support vector machine (SVM) to identify the target area. Also a fuzzy control model was analyzed and exerted to compensate the nonlinearity and hysteresis of the variable rate spraying system. Experimental results proved that the approach was applicable to reducing the amount of pesticides during UAV spraying, which can provide a reference for precision agriculture aviation in the future.

Keywords: vision sensor, UAV, adaptive spray, variable rate spraying, fuzzy control, empty area, precision agriculture aviation DOI: $10.25165 /$ j.ijabe.20191203.4358
\end{abstract}

Citation: Wang L H, Lan Y B, Yue X J, Ling K J, Cen Z Z, Cheng Z Y, et al. Vision-based adaptive variable rate spraying approach for unmanned aerial vehicles. Int J Agric \& Biol Eng, 2019; 12(3): 18-26.

\section{Introduction}

Chemical control is one of the most important production management techniques in rice production. Since the 1950s, in order to overcome the shortcomings of common spraying approach of pesticide, the researchers had carried out a large number of research about spraying pesticides while developed plant protection machinery suitable for rice field operations ${ }^{[1,2]}$. Recently years, it is gradually realized that ground crop protection spray machines are constrained by many factors including topography and later stage of crop growth, and have poor field adaptability and operational results ${ }^{[3]}$. As a result, agricultural aviation has become popular worldwide. However, it is common that the

\section{Received date: 2018-04-28 Accepted date: 2019-02-27}

Biographies: Linhui Wang, $\mathrm{PhD}$, research interests: agricultural aviation application, Email: Jetwlh@stu.scau.edu.cn; Yubin Lan, PhD, Professor, research interest: agricultural aviation application, Email: ylan@scau.edu.cn; Kangjie Lin, postgraduate, research interest: Machine learning, Email: lingkangjie@foxmail.com; Zhenzhao Cen, postgraduate, research interest: agricultural aviation, Email: 641086930@qq.com; Ziyao Cheng, postgraduate, research interest: agricultural aviation, Email: 472137237@qq.com; Yongxin Liu, $\mathrm{PhD}$, research interest: agricultural aviation, Email: 229568069@qq.com; Jian Wang, PhD, research interest: agricultural aviation, Email: 2206669185@qq.com.

*Corresponding author: Xuejun Yue, PhD, Professor, research interests: agricultural aviation application. Room 103, College of Electronic Engineering, South China Agricultural University, Guangzhou 510642, China. Tel: +86-13802969638, Email: yuexuejun@scau.edu.cn. excessive pesticides will not only bring rising costs but also result in environmental damage for farmers ${ }^{[4,5]}$. Today, researchers in the world have conducted numerous studies on how to improve droplet deposition results and pesticide usage in precision agricultural aviation. Adaptive and variable rate spraying is a focus research field about pesticide use reduction among these trends ${ }^{[6,7]}$

There is a common phenomenon in rice planting management stage that some empty areas, which consist of water and a small amount of weeds, are randomly located in farmland. That is mainly due to human factors such as uneven planting, missed sowing and excessive fertilization, or natural disasters such as pests, floods, typhoons, etc., and then cause plants necrosis to form empty area. However, the common UAV spraying system is not applicable to spray pesticide on this farmland for uniform spray, which makes it difficult to reduce the use of pesticides.

With the development of vision sensor technology, it is of great significance in making plant protection machinery smarter. Therefore, it is a good way to apply vision sensor to UAV spraying system, where it can identify farmland conditions automatically and then control the amount of pesticides especially for working in the farmland which has empty area.

In this paper, a novel adaptive variable rate spraying approach was proposed based on vision sensor for the empty area of rice fields. Among this approach, the image was first preprocessed with Lucy-Richardson algorithm, and then the target area was split from the background with $k$-means for feature extraction. Finally 
a SVM model was built to identify empty areas. Additionally, an adaptive control strategy was proposed that makes the flow rate changes with the area of the empty area. In order to improve the stability of variable rate spraying system, this study analyzes and exerts a fuzzy control model, which is expected to provide a measures for pesticides reduction in precision agricultural aviation.

The major contributions of this research work are highlighted as below:

1) To develop an adaptive variable rate spraying system which is suitable for rice fields. The spraying system consists of a visual sensing system and a flow control system. It could automatically adjust the amount of pesticide sprayed to the area of empty areas real time.

2) To develop a novel approach to identify the empty areas from the image of rice field. In this approach, the image is firstly preprocessed by Lucy-Richardson algorithm, and then separated the target area from the background by $k$-means for feature extraction. Finally, the characteristic parameters are filtered out by positive contribution, and as the input parameters of the SVM model to identify the target area.

3) To analyze and exert a fuzzy control model to improve the nonlinearity and hysteresis of variable rate spraying system.

\section{Related works}

Pesticide spraying is one of the most significant approaches that are utilized to protect plants while implementing prevention for diseases and plague of insects. At present, the UAV equipped with a spraying system has been investigated more than before and has been used for pesticide applications in the world ${ }^{[8,9]}$.

Many researchers are exploring how to design a better spraying system and implement it on the UAV. Huang et al. ${ }^{[10]}$ developed an electrostatic spraying system for UAV, and their field test results showed that charged droplets could reduce $50 \%-70 \%$ of the pesticide waste. $\mathrm{Xu}$ et al. ${ }^{[11]}$ used the PWM technology to design a UAV variable rate spraying system to control pesticide spraying by adjusting the duty cycle. $\mathrm{Ru}$ et al. ${ }^{[12]}$ designed an electrostatic spraying device based on a cone-shaped spraying nozzle and installed it on a UAV for field tests. The results showed that the electrostatic spraying increased the average droplet coverage at the canopy, middle, and bottom of a target plant by 35.4, 26, and 9 droplets $/ \mathrm{cm}^{2}$, respectively. Zhang et al. ${ }^{[13]}$ designed a UAV electrostatic spraying system, and the field test results showed that compared with the non-electrostatic spraying device, their system improved the average deposition density by $13.6 \%$ and $32.6 \%$ in the middle. However, the approaches described above cannot effectively decrease the use of pesticides, because their UAV spraying systems are using a uniform spraying method.

A small number of scholars turned their eyes to new research directions. They thought that adaptive variable spray which applies the required amount of pesticides to the target objects by obtaining the parameters of the machine status, morphology, location of pests and diseases, crop density and area, can play a great significant role in cost saving and environmental pollution reduction. Shahemabadi et al. ${ }^{[14]}$ studied a variable spray control algorithm based on GPS speed change. They adjusted flow rate by changing the number of pulses per second and pulse width. However, the update frequency of the GPS data in this system was too slow, which made this system unsuitable for high-speed spraying with UAV. Wang et al. ${ }^{[15]}$ designed a UAV-based variable rate spraying system, which automatically adjusts pump's pressure to match the UAV's flight speed. But they didn't conduct further research on the influence of the control algorithm on droplet deposition.

Some researchers believed that UAV remote sensing provides a non-destructive and cost-effective platform for rapid monitoring of weeds, pests, and diseases by converting the visual data into a prescription map through image processing which will be utilized by the spraying system to carry out variable rate spraying. José Manuel Peña et al. ${ }^{[16]}$ developed a robust and automated object-based image analysis (OBIA) procedure for automatic discrimination of crop rows and weeds in georeferenced and $2-\mathrm{cm}$ spatial resolution remote images. Their algorithm effectively identified all the crop rows based on their linear pattern and the contextual features of the vegetation objects belonging to the rows. Using the OBIA method requires feature selection which must be performed by manual designs. Although hand-designed features are a proven approach, they vary based on applications and are hard to be generalized ${ }^{[17]}$. Therefore, UAV remote sensing cannot assure a steady location, height, and acquisition time, and its reaction speed is slow. And how the remote data guides the UAV variable rate spraying system remains to be a difficulty.

Someone thought that real-time image processing could bridge the gap between remote sensing and UAV variable rate spraying system $^{[4]}$. With the development of computer vision technology, SVM is gradually used to process images in real time. Its applications in the fields of pest identification, crop classification, soil spectral detection and yield prediction had studied ${ }^{[18-21]}$. Tian et al. ${ }^{[22]}$ combined SVM with various techniques such as median filtering, statistical pattern recognition, and morphology to extract feature parameters and established a SVM grape disease identification model with good stability. Camargo et al. ${ }^{[23]}$ used support vector machines to study the plant image recognition problem based on 52 feature parameters such as shape, color, and texture. The results showed that the SVM had poor recognition rate regarding a single feature parameter, while multi-feature recognition performed well with an optimal recognition rate up to $90 \%$. The above studies proved that the SVM is an accessible method to classify and identify target images with multiple features, especially for targets with various colors and texture features.

From the above review, it is concluded that research on adaptive variable rate spraying is still insufficient although UAV is becoming a trend in agricultural plant protection. Moreover, some challenges in the application of real-time image processing with UAV adaptive variable rate spraying system, and no related research for rice field empty area identification has been reported yet.

\section{Materials and methods}

\subsection{UAV and vision-based spraying system}

The vision-based spraying system consists of an image acquisition unit and a spraying structure, as shown in Figure 1. The image acquisition unit is made up of a Charge-Coupled Device (CCD) camera (SONY SSC-G813), a data buffer memory, and a Graphics Processing Unit (GPU) embedded device (NVIDIA Jetson TX2). The spraying structure is composed of a Pulse Width Modulation (PWM) module, medicine box, diaphragm pump, flow sensor, relay and centrifugal nozzle. Figure 1a clearly shows the spraying system's mechanical structure on UAV. The CCD camera is located at the right slide rail while the centrifugal nozzle at the left slide rail. And the right slide rail represents the front of UAV. The CCD camera can adjust the position of slider to the optimal delay distance, which ensures a sufficient response time of the software and hardware. 


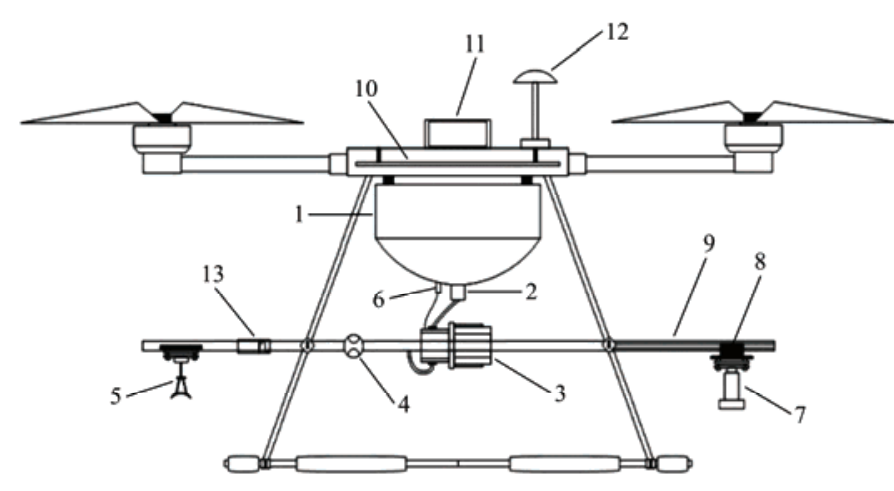

a. System mechanical structure

Spraying tank 2. Filter 3. Water pump 4. Flow 10. Processor 11. Power 12. GPS 13. Relay

Figure 1 Structure of vision-based spraying system on UAV

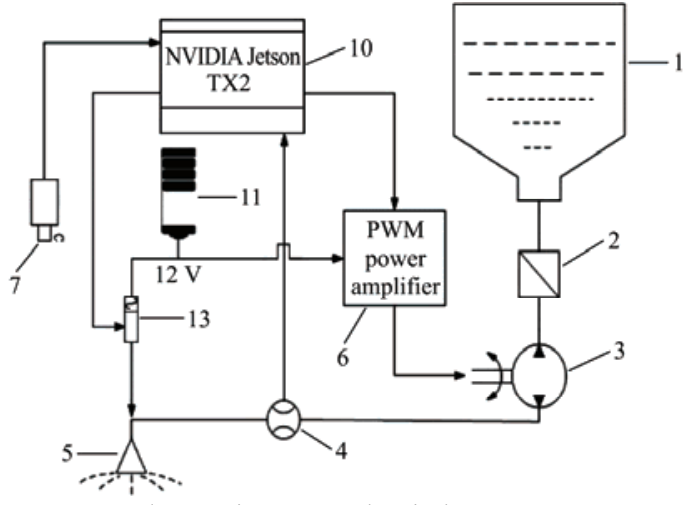

b. Spraying system electrical structure

\subsection{Proposed approach for empty area identification}

A novel empty area identification approach is provided in Figure 2. We collected the data of empty area images captured by the UAV in rice field at an acquired rate of $30 \mathrm{fps}$ and effective $750 \times 580$ pixels of single frame. By this approach, we obtained many image frames with a resolution of $375 \times 290$ pixels by reducing half pixels in every single-frame image. Then we selected 200 sample images from them, among which 150 images were for training and 50 images were for prediction. The Lucy-Richardson algorithm was pretreated on each image to obtain a clear image, which could be used to reduce dimensions with principal component analysis (PCA) later. Then, a clear image was separated from the image of the empty area by the $k$-means algorithm. Next, the color and texture features were extracted with color matrix and gray-level co-occurrence matrix, respectively. Finally, single feature parameter, combination of different empty area's feature parameters, and total feature parameters were merged into the input parameters of the SVM to build the classifier. Genetic algorithm (GA) optimization was performed at the same time. A stepwise discrimination method was used to test the contribution of each feature parameter in order to obtain the optimal feature parameter combination and an empty area image recognition model with the optimal recognition rate.
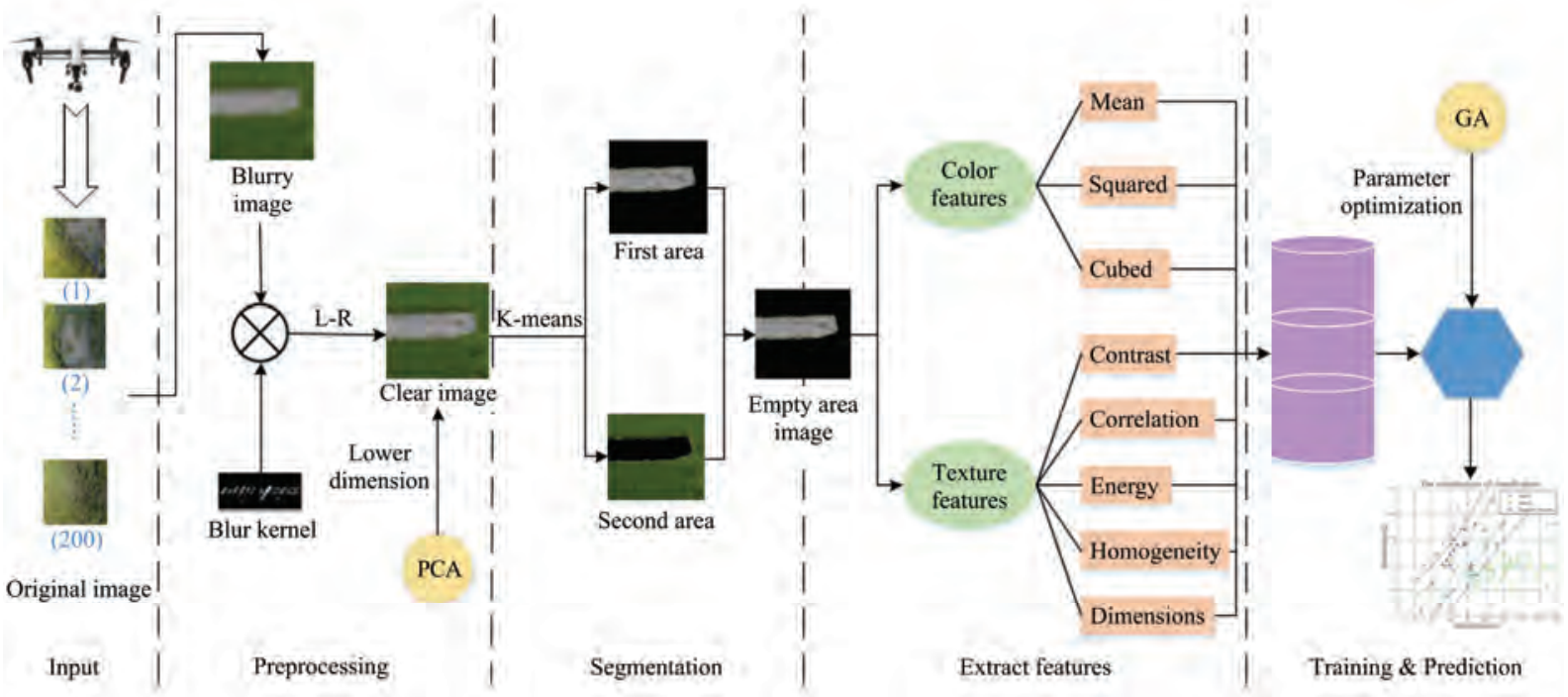

Figure 2 The flowchart of our approach

\subsubsection{Lucy-Richardson algorithm}

In image acquisition, due to mechanical vibration, slanting sloshing, and camera motions, the captured images are blurry with low contrast and unclear texture. In order to improve the image quality, we applied the Lucy-Richardson algorithm to the image sets. The Lucy-Richardson model can be described as:

$$
Y=\Lambda \times P
$$

where, $Y$ is the degenerated image; $\Lambda$ is the original image; $P$ is a point spread function, and * represents a convolution operation. The restoration function which comes from Poisson distribution and iterative derivation ${ }^{[24]}$ as Equation (2):

$$
\lambda_{i}^{(t+1)}=\lambda_{i}^{(t)} \sum_{j=1}^{d} \frac{y_{j} p(i, j)}{\sum_{k} \lambda_{k}^{(t)} p(k, j)}
$$

\subsection{2 $\mathrm{K}$-means algorithm}

It is necessary to separate both the crop areas and empty areas in the field aerial images for feature extraction in later steps. Compared with common edge detection algorithms, the $k$-means algorithm has higher accuracy in obtaining edge pixels, and can be used to realize segmentation of crop areas and empty areas ${ }^{[25]}$. The steps below show how to carry out the $k$-means:

1) Initialize the centroid of the distance between 2 clusters (generated randomly).

2) Calculate the Euclidean distance between all the samples and each centroid, and add the data sample to the cluster with the shortest Euclidean distance (record the number of the data sample).

3) Calculate the centroid of each cluster and update it. If the new centroid is equal to the original centroid, the iteration ends. 
Otherwise, it will go back to step 2 and the iteration will continue.

3.2.3 Feature extraction

It is difficult to identify all the objects by shape feature parameters due to the empty areas' irregular shape. Thus, only 22 feature parameters which consist of 9 color feature parameters and 13 texture feature parameters are extracted.

1) Color features are extracted by color torch

Color moments are an effective color feature, of which the useful information mainly concentrates on the low-order moments ${ }^{[26]}$. In this paper, the first and third moments, which are named by mean $\mu$, squared difference $\sigma^{2}$, and cubic difference $\varepsilon^{3}$ in $\mathrm{R}, \mathrm{G}$, and B channels of the color image, respectively, are used as the color feature parameters, and thus there are 9 color feature parameters in total. Their equations are as follows:

$$
\begin{aligned}
\mu & =\frac{1}{p} \sum_{j=1}^{p} f_{j} p\left(f_{j}\right) \\
\sigma^{2} & =\frac{1}{p} \sum_{j=1}^{p} f_{j} p\left(f_{j}-\mu\right)^{2} \\
\varepsilon^{3} & =\frac{1}{p} \sum_{j=1}^{p} f_{j} p\left(f_{j}-\mu\right)^{3}
\end{aligned}
$$

where, $P$ is the total pixels of the empty area; $f_{j}$ represents any coordinate in the empty area, and $p\left(f_{j}\right)$ represents the color value of point $f_{j}$.

2) Texture features are extracted by gray level co-occurrence matrix

Gray level co-occurrence matrix is an effective method to analyze texture features ${ }^{[27]}$. In this research work, twelve texture features which make up the contrast (indicates pix at the direction of $\left.0^{\circ}\right)$, the correlation $\left(45^{\circ}\right)$, the energy $\left(90^{\circ}\right)$, and the homogeneity $\left(135^{\circ}\right)$ in the gray level co-occurrence matrix of R, G, and B channels, respectively, are extracted. The four feature parameters represent horizontal, vertical, diagonal, and anti-diagonal directions, respectively. Additionally, the $4 \times 4$ box fractal dimension of binary image is also selected as a texture feature parameter of the empty area image.

\subsubsection{SVM algorithm}

Assuming that there are a large number of image features in the empty area, the optimal image classification function with quadratic programming as Equation (6):

$$
f(x)=\operatorname{sgn}\left\{\sum_{i=1}^{n} a_{i}^{*} y_{i}\left(x_{1} \cdot x\right)+b^{*}\right\}
$$

where, sgn represents symbolic functions; $a^{*}=\left(a_{1}^{*}, \ldots, a_{n}^{*}\right)$ is Lagrange multiplier; $b^{*}$ is a threshold. The classification function of the empty area image can be optimized to Equation (7).

$$
f(x)=\sum_{i=1}^{n} a_{i}^{*} y_{i} K\left(x_{1} \cdot x\right)+b^{*}
$$

where, $K$ is kernel function. Many test results have showed that the Radial basis function (RBF) is the best kernel function when it is applied to the samples, so the RBF was selected as the kernel function of the $\mathrm{SVM}^{[28]}$.

$$
K(x, y)=\exp \left\{-\frac{|x-y|^{2}}{\sigma^{2}}\right\}
$$

\subsection{Establishing the Variable rate spraying Decision Model}

Two problems have to be solved in the variable rate spraying process, how to reduce errors caused by the system time delay and how to design the control strategy and improve the robustness of the spraying system under non-linear conditions. In order to tackle the preceding problems, a variable rate spraying decision model was established, as shown in Figure 3.

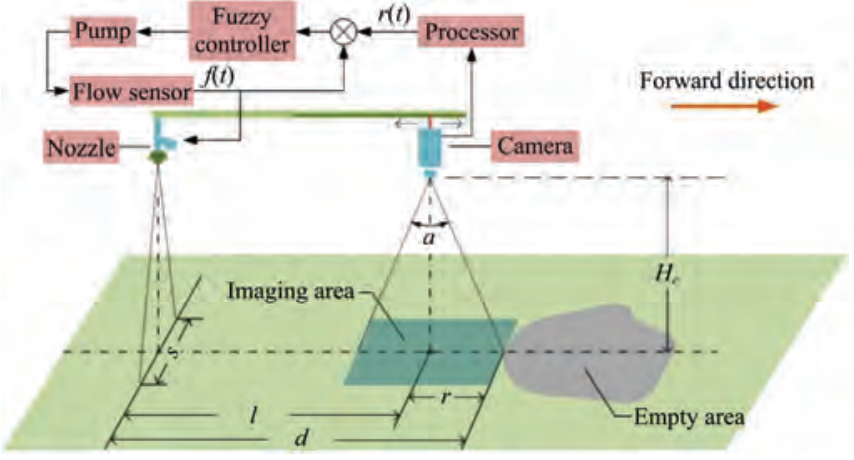

Figure 3 Variable spray decision model

The key solution for the first problem is to calculate $l$ which is the installation distance between the nozzle and the camera. Where, $r$ is the distance from the camera to the edge of the empty area. And if the height of the camera is fixed, $r$ will be a constant. Equation (9) is obtained with the trigonometric function:

$$
r=H_{c} \tan \frac{\alpha}{2}=0.4 H_{c}
$$

where, $H_{c}$ is the height between the camera and the crop canopy, and $\alpha$, which is equal to $45^{\circ}$, is the imaging angle of the camera.

In addition, there is a delay distance $d$ between the nozzle forehead and the edge of the empty area, which is a key parameter that we need to calculate. Obviously, it only relates to the UAV's flight speed $\mathrm{v}$ and the response time of the system's hardware and software $t_{c}$, which is a time delay period from image acquisition to centrifugal nozzle powering on.

$$
d=v t_{c}
$$

Thus, we can get the ideal mounting pitch $l$ that can be seen in Equation (11) after substituting Equations (9) and (10) into $d=(l+r)$, and then adjust it with the slider above the camera.

$$
l=v t_{c}-0.4 H_{c}
$$

Figure 3 clearly shows that, firstly, the empty area image is collected by the camera, which is the input of the processor that analyzes the image data with the SVM model and outputs the ideal amount of spray $r(t)$ is optimized by the fuzzy controller and operates as the input of the pump for the first time. Finally, it is converted to $f(t)$, which is measured by the flow sensor, and enters the fuzzy controller again until the relative error can be neglected.

Figure 4 shows the schematic diagram of the operation when $\mathrm{UAV}$ approaches or leaves the empty area during spraying.

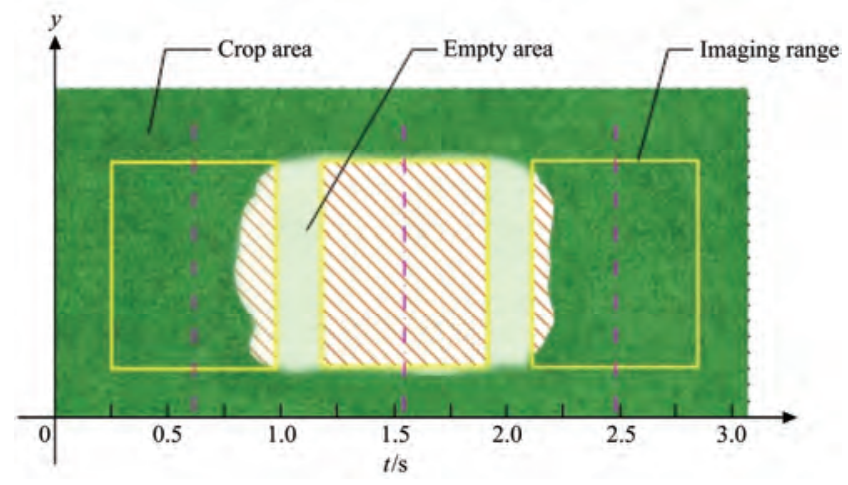

Figure 4 Schematic of spray test

When the total area of the imaging area is defined as $S_{c}$ (yellow rectangular frame) and the size of empty area identified in the imaging range is defined as $S_{e}$ (red stripe area), the ratio $p$ of them can be defined as:

$$
p=\frac{S_{e}}{S_{c}} \times 100 \%, p \in[0,100 \%]
$$


The empty area image which is generated by using the $k$-means algorithm and only consists of empty area pixel $(>0)$ and non-empty area pixel $(=0)$, is identified by the SVM. Thus, $S_{e}$ can be indicated by the total number of the empty area pixels ${ }^{[29]}$.

$$
s_{e}=\sum_{i=0}^{m} \sum_{j=0}^{n} \lambda(i, j)
$$

where, $m$ is the horizontal resolution of the image; $n$ is the vertical resolution of the image; $\lambda(i, j)$ indicates that the pixel of $(i, j)$ belongs to the empty area pixels and equals to 0 . Substituting $s_{c}=m \times n$ and Equation (13) into Equation (12), and then getting the Equation (14):

$$
p=\frac{\sum_{i=0}^{m} \sum_{j=0}^{n} \lambda(i, j)}{m \cdot n} \times 100 \%, p \in[0,100 \%]
$$

In this paper, $x$ is defined as the PWM duty cycle. When the UAV steadily moves to the empty area, $p$ will increase gradually. As a result, the system will decrease $\mathrm{x}$ to slow down the motor speed of the diaphragm pump and the nozzle will output a small amount of spray further. If the UAV leaves the empty area, the amount of spray will reduce. According to the test results, if the frequency of the PWM's control signal is set to $10 \mathrm{~Hz}$, and $x$ is from $45 \%$ to $100 \%$, the flow rate will stay at $3.3 \mathrm{~L} / \mathrm{min}$. When $x$ is lower than $7 \%$, the diaphragm will be unable to work normally. The polynomial relationship model is based on the fitting duty cycle $x$ and spraying flow rate $Q$, as shown in Figure 5 .

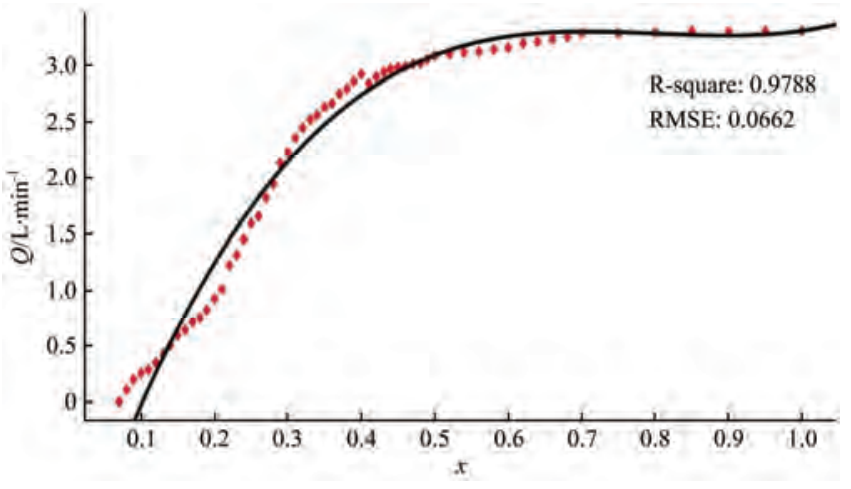

Figure 5 Polynomial model

where, $R^{2}$ is 0.9788 , and RMSE is 0.0662 , which indicates that the model has a good linear correlation. Its function is defined as:

$$
Q=\left\{\begin{array}{l}
0, x \in[0,0.07] \\
60.46 x^{3}-80.59 x^{2}+36.99 x-2.63, x \in(0.07,0.45) \\
3.3, x \in[0.45,1]
\end{array}\right.
$$

Since it can be seen that the duty cycle $x$ and the ratio $P$ are in a linear relationship, the function relationship can be set as Equation (16):

$$
x=-0.38 p+0.45
$$

Finally, substituting Equation (15) into Equation (14), and then getting Equation (17) about $Q$ and $P$. But note that $Q$ is equal to the above mentioned $r(t)$.

$$
Q=\left\{\begin{array}{l}
3.3, p=0 \\
-3.26 p^{3}+0.21 p^{2}-0.56 p+3.31, x \in(0,100 \%) \\
0, p=100 \%
\end{array}\right.
$$

\subsection{Fuzzy control modeling analysis}

The system uses the Plane 12V45W PLD-1206 type diaphragm pump, of which the maximum flow rate is $3.5 \mathrm{~L} / \mathrm{min}$. The control system is composed of the PWM power amplifier, motor, transmission system, and diaphragm. Its structure is shown in Figure 6.

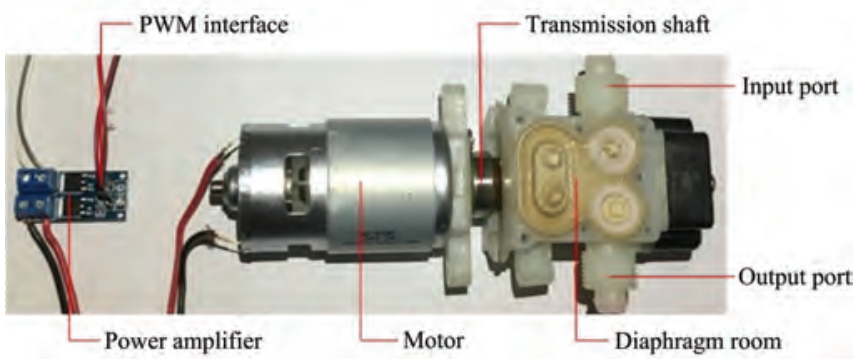

Figure 6 Diaphragm pump structure diagram

The PWM power amplifier and the motor in the flow control system function as a unit, which is equivalent to an inertial link, and their gain coefficient is set to $K_{1}$. Other factors of the flow control system, such as the piston movement of the transmission shaft and the diaphragm and the flow of liquids, are considered as a whole, and their gain factor is set to $K_{2}$. According to the actual operation status of the flow control system, the time of the power amplifier and the motor is set to 0.02 , the inertial time is set to 1 , the pure system delay time is set to 1.5 , and the system's total gain $K_{1} K_{2}$ equals to 1.6. Thus, the model of the flow control system's transfer function is given as:

$$
G(s)=\frac{1.6 \exp (-1.5 s)}{0.02 s^{2}+1.02 s+1}
$$

Figure 7 shows the fuzzy controller designed for the flow control system's hardware. The deviation between the target flow $r(t)$ and the actually measured variable $f(t)$ after the analog-to-digital conversion is used as the input $e$ and differentiates with the previous deviation to obtain the deviation changed $e_{c}$. The motor rotation speed is the controller output $u$.

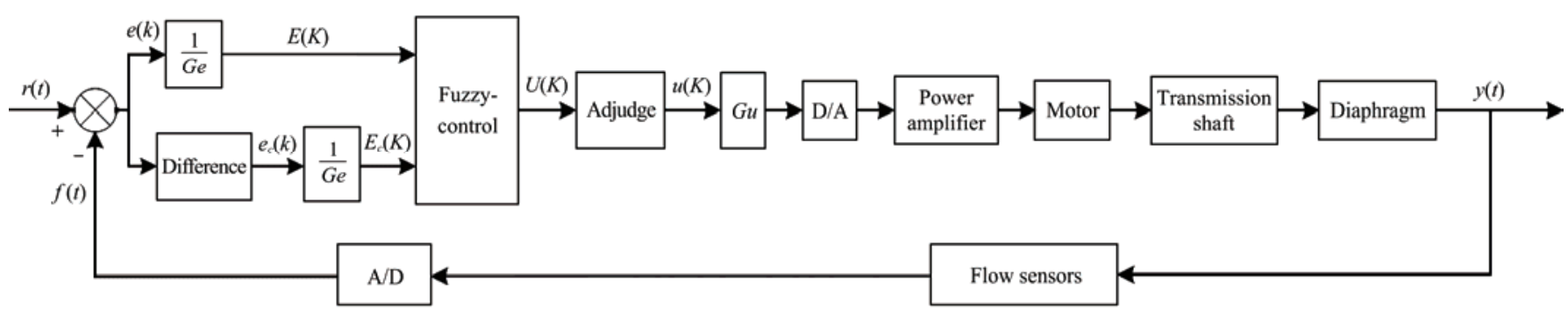

Figure 7 Fuzzy controller schematic

The deviation e and the deviation change $e_{c}$ are processed by the fuzzy controller to obtain the output $u$. Then the digital-to-analog converter converts the digital signal into an analog signal and sends it to the PWM power amplifier, changes the input voltage of the motor, adjusts the rotation speed, and then drives the diaphragm to make piston movements through the transmission system to control the flow. The flow rate sensor is used to detect the flow of the pump, and the deviation between the measured flow and the target flow is continuously compared and corrected to improve the control accuracy.

The fuzzy variables corresponding to $e, e_{c}$, and $u$ are $E, E_{c}$, and $U$, respectively. The fuzzy states of the three variables are 
selected as:

$E=\{N B, N M, N S, N Z, Z, P S, P Z, P M, P B\}$

$\mathrm{Ec}=\{\mathrm{NB}, \mathrm{NM}, \mathrm{NS}, \mathrm{Z}, \mathrm{PS}, \mathrm{PM}, \mathrm{PB}\}$;

$\mathrm{U}=\{\mathrm{NB}, \mathrm{NM}, \mathrm{NS}, \mathrm{Z}, \mathrm{PS}, \mathrm{PM}, \mathrm{PB}\}$.

Each variable selects a triangular membership function, as shown in Figure 8. Due to liquid turbulence, the flow rate is a relatively unstable quantity and fluctuations of the flow rate value may be detected each time. To ensure the basic stability of the flow, when $E$ is allowed to have a small deviation within the acceptable range of the spraying concentration error, the membership function of $E$ needs to be sparse in the middle and become dense at both ends. In order to make rapid responses when the deviation is large, the response accuracy should be improved and the amount of overshoot should be reduced when the deviation is small. Therefore, the membership function of the output volume $U$ is set to be sparse at both ends and dense in the middle ${ }^{[30]}$
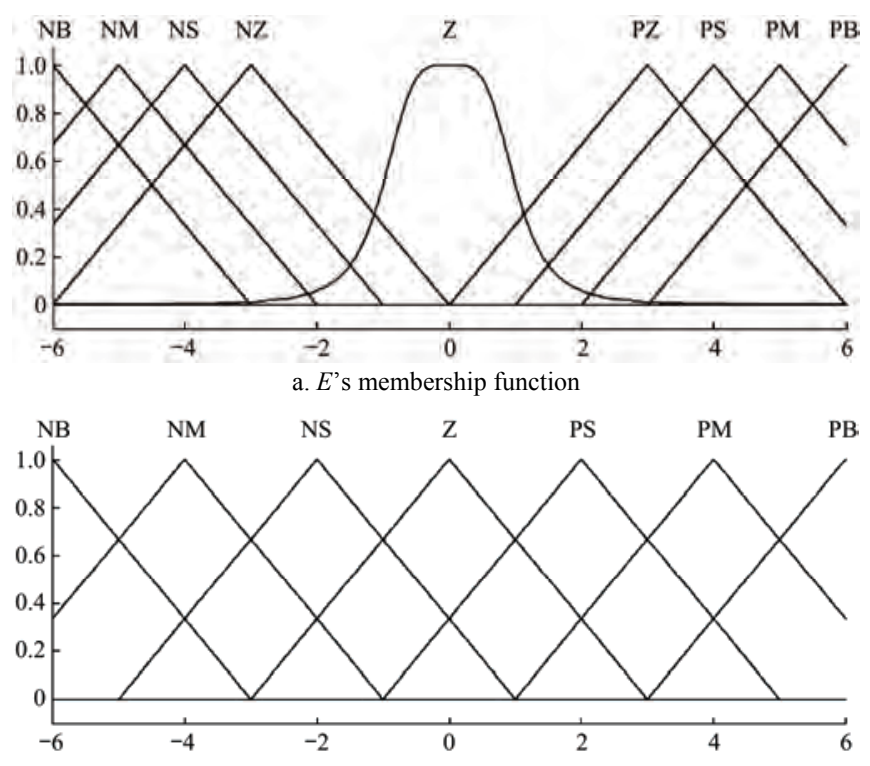

b. $E_{c}$ 's membership function

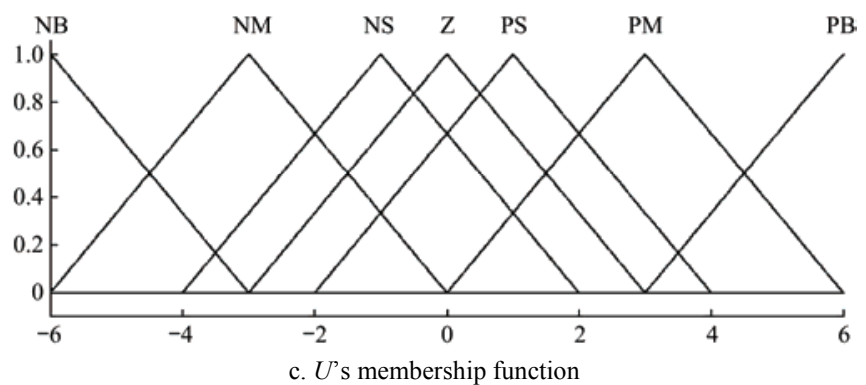

Figure 8 Membership functions of $E, E_{c}$ and $U$

According to expert theory and practical experiences, fuzzy conditional inference "if $\mathrm{A}$ and $\mathrm{B}$, then $\mathrm{C}$ " is used to make the fuzzy control rules shown in Table 1 .

Table 1 Rules of fuzzy control

\begin{tabular}{cccccccccc}
\hline$U$ & $E$ & & & & & & & & \\
$E_{c}$ & NB & NM & NS & NZ & Z & PZ & PS & PM & PB \\
\hline NB & PB & PB & PB & PB & PM & PM & PS & Z & Z \\
NM & PB & PB & PM & PM & PS & PS & Z & Z & NS \\
NS & PB & PM & PM & PS & Z & Z & Z & NS & NS \\
Z & PB & PS & PS & Z & Z & Z & NS & NS & $/$ \\
PS & PM & PS & Z & Z & Z & NS & NS & NM & $/$ \\
PM & PS & Z & Z & NS & NS & NS & NM & NB & NB \\
PB & Z & Z & NS & NM & NM & NB & NB & NB & NB \\
\hline
\end{tabular}

\section{Results and analysis}

\subsection{Data set preprocessing results}

A clear image come from the motion blur image which is processed by the Lucy-Richardson algorithm. The both of them R, G, B pixels' distribution are shown in Figure 9.

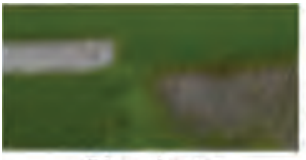

Blurry image

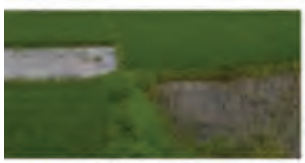

Clear image

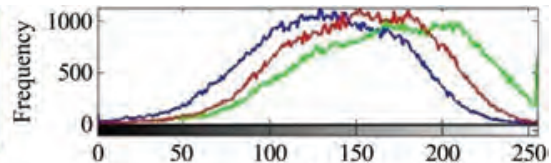

Gray leves

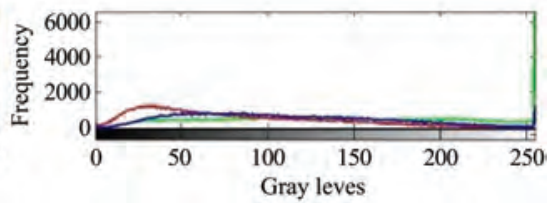

Figure 9 Original image and clear image and they R, G, B pixel distribution

In the Figure 9, the overall brightness is high while the contrast and sharpness are low due to the high frequency and concentration of the high-brightness pixels. Comparing with the former image, the quality of the image processed by the Lucy-Richardson algorithm is higher, the color degradation is corrected, and the pixel distribution of its RGB channels histogram is balance and disperse.

The $k$-means hard clustering algorithm is used to segment a clear image. The result is shown in Figure 10.
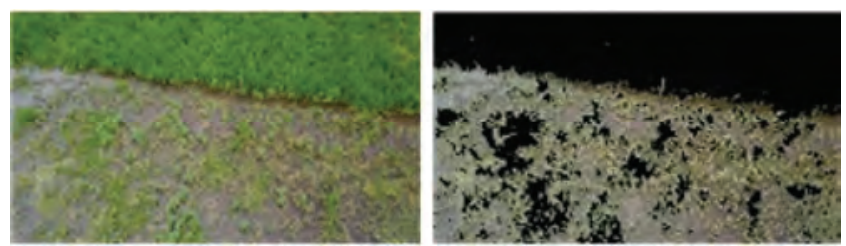

Figure 10 Results of image segmentation of farmland aerial image

In Figure 10, the algorithm could accurately segment the empty areas from the image. The obtained segmentation image which was to be used for feature extraction later was ideal

\subsection{Effective feature selection and SVM model simulation analysis}

The color feature and texture feature results are shown in Table 2 and Table 3, respectively. The values in the tables indicate the ratio of the training sample recognition rate to the test sample recognition rate. If the ratio is greater than 1 , it means that the feature parameter has a positive contribution; if it is equal to 1 , no contribution is made, or there is a negative contribution.

In Table 2 and Table 3, feature recognition rate and combination feature parameter vary. Third moment's combinations have the best recognition effect in color feature, and the correlation's combinations are the best in texture feature. The negative contribution to the recognition result includes the first moment of the $\mathrm{G}$, the second moment of B in color feature, the correlation and homogeneity of the R, and the energy of $\mathrm{B}$ in texture feature. All of these will be removed and not be input to the SVM.

A total of 17 best feature parameters were selected from Table 2 and Table 3 with a positive contribution as the input of the SVM, and trained 150 training samples to obtain the optimal hyperplane, as shown in Figure 11.

In Figure 11, the middle purple line is the optimal hyperplane. It can identify empty areas and crop areas and deliver an ideal recognition effect. During the training process, the GA function 
found the optimal parameter $c=50.64, g=11.5103$.

Three different types of samples $(750 \times 580$ pixels, $580 \times$ 320 pixels, and $375 \times 290$ pixels) were selected to test for verifying the performance of the SVM model. The number of each type of samples was 50. Its recognition rate and response time were recorded, and the results were shown in Figure 12.

Table 2 Farmland aerial image color feature recognition preferred results

\begin{tabular}{ccccc}
\hline \multirow{2}{*}{$\begin{array}{c}\text { Color features } \\
\text { parameter }\end{array}$} & $\mathrm{R}$ & Single recognition rate/\% & Combination recognition rate/\% \\
\cline { 2 - 4 } & $\mathrm{R}$ & $\mathrm{G}$ & $\mathrm{T}$ & \\
\hline First moment & $82.00 / 80.32$ & $73.75 / 80.31$ & $77.5 / 76.19$ & $78.44 / 73.43$ \\
Second moment & $79.5 / 71.20$ & $85.55 / 80.45$ & $80.74 / 82.55$ & $71.57 / 70.46$ \\
Third moment & $80.00 / 78.90$ & $79.00 / 74.32$ & $75.00 / 71.58$ & $86.00 / 76.50$ \\
\hline
\end{tabular}

Table 3 Farmland aerial image texture feature recognition preferred Results

\begin{tabular}{ccccc}
\hline \multirow{2}{*}{$\begin{array}{c}\text { Texture features } \\
\text { parameter }\end{array}$} & \multicolumn{3}{c}{ Single recognition rate/\% } & Combination recognition rate/\% \\
\cline { 2 - 4 } & $\mathrm{R}$ & $\mathrm{G}$ & $\mathrm{B}$ & $78.24 / 74.50$ \\
Contrast & $78.56 / 77.32$ & $78.53 / 77.32$ & $76.71 / 73.95$ & $84.68 / 82.46$ \\
Correlation & $75.00 / 78.54$ & $84.56 / 83.43$ & $83.00 / 80.43$ & $78.52 / 69.38$ \\
energy & $68.78 / 62.50$ & $69.67 / 62.63$ & $60.52 / 65.66$ & $77.53 / 75.59$ \\
Homogeneity & $87.46 / 79.54$ & $78.55 / 75.58$ & $86.13 / 81.96$ & $78.44 / 70.00$ \\
Fractal dimension & & $78.44 / 70.00$ & & $78.94 / 75.50$ \\
\hline
\end{tabular}

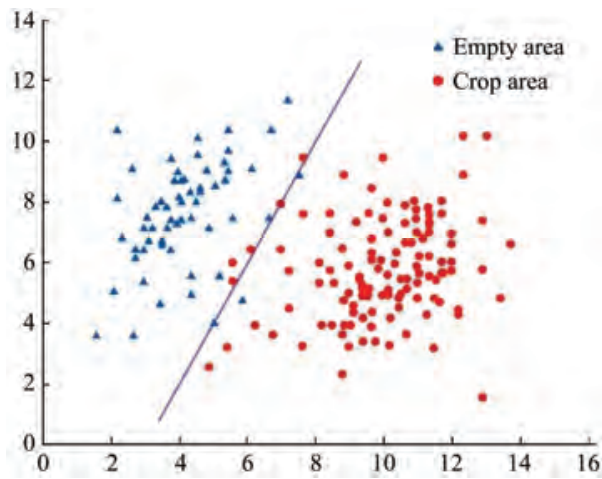

Figure 11 Optimal hyper plane after training

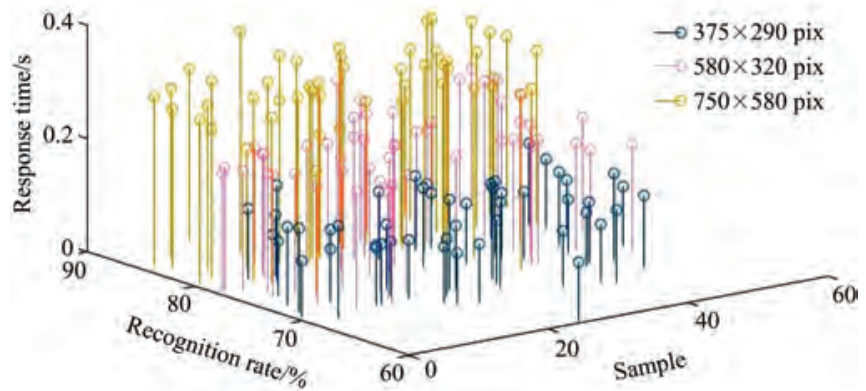

Figure 12 Recognition rate and response time of three types pixel samples

In Figure 12, the average recognition rate of the $375 \times 290$ samples is $72.69 \%$. When the sample's pixels increase, the average recognition rate increases correspondingly. The reason is that with the increase of the pixels during model recognition, the image structure information becomes more complete and it's easy to extract effective feature parameters. But more calculation is required, resulting in a greatly increased response time for the algorithm. For example, the average response time of the $750 \times 580$ samples reached $0.299 \mathrm{~s}$. Therefore, considering the real-time response requirement of the embedded system ${ }^{[31,32]}$, an image with less pixels should be selected as far as the relatively slow recognition rate is tolerated.

\subsection{Adaptive variable rate spraying experiments with UAV}

Agricultural Training Station of the South China Agricultural University located in Guangzhou, Guangdong, China, was selected as the experimental site, and rice was at the booting stage. The established SVM model, transfer function, and fuzzy control rules were transplanted to the on-board spraying system. During the UAV operation process, set $H_{c}$ is set to $1.5 \mathrm{~m}$, and $v$ to $3 \mathrm{~m} / \mathrm{s}$. After the test, $t_{c}$ took the average response time of the system as $0.45 \mathrm{~s}$. From Equation (10), it could be seen that the installation distance between the camera and the nozzle was equal to $0.73 \mathrm{~m}$.

\subsubsection{Spraying effect detection}

An empty area on the UAV's planned flight path was selected, as shown in Figure 13. Water sensitive papers were placed at positions $\mathrm{A} 1, \mathrm{~A} 2, \mathrm{~B} 1, \mathrm{~B} 2, \mathrm{C} 1$, and $\mathrm{C} 2$. The spraying width was larger than the water sensitive paper spacing on both sides. The UAV's operation direction was indicated by the yellow arrow.

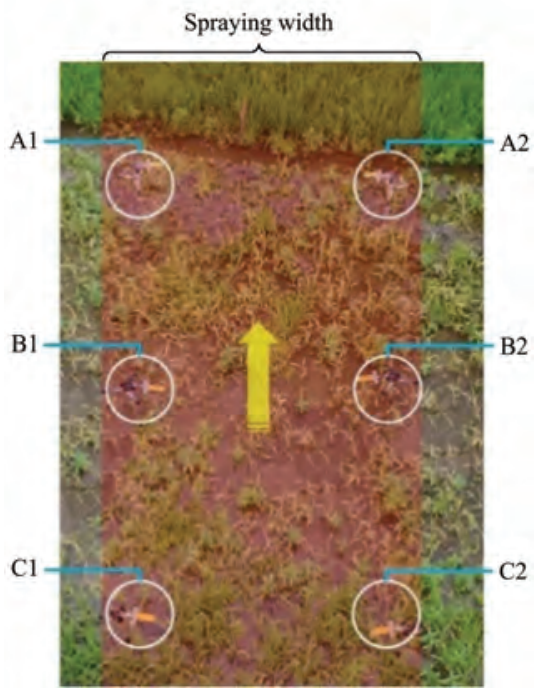

Figure 13 Spray effect test

As shown in Figure 14, water sensitive papers deployed at different sites were scanned in sequence, where the black spots were the areas sprayed by the UAV while the yellow areas were non-sprayed areas.

Using Depositscan software (http://www.ars.usda.gov/ research/software/download/?softwareid=247) to process the water sensitive paper images of $\mathrm{A} 1-\mathrm{C} 2$, and analyzing $P$, droplet coverage, and droplet diameter variation coefficient. The result is shown in Table 4. 


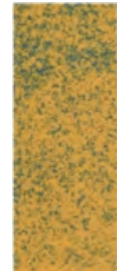

A1

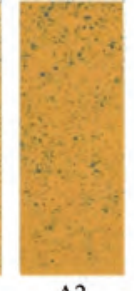

A2

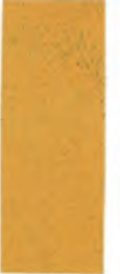

B1

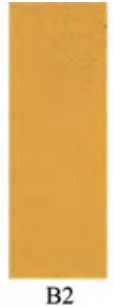

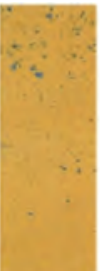

$\mathrm{Cl}$

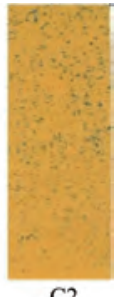

$\mathrm{C} 2$
Figure 14 Water sensitive paper deployed at different site

Table 4 Droplet deposition results

\begin{tabular}{ccccc}
\hline Site & $\mathrm{P} \%$ & Coverage $\%$ & Mean coverage \% & CV of droplet diameter \\
\hline $\mathrm{A} 1$ & \multirow{2}{*}{72.7} & 4.89 & 4.47 & 0.146 \\
$\mathrm{~A} 2$ & & 4.05 & & 0.151 \\
\hline $\mathrm{B} 1$ & \multirow{2}{*}{94.5} & 0.95 & 0.89 & 0.960 \\
$\mathrm{~B} 2$ & & 0.83 & & 0.841 \\
\hline $\mathrm{C} 1$ & \multirow{2}{*}{83.0} & 1.12 & 1.13 & 0.373 \\
$\mathrm{C} 2$ & & 1.14 & & 0.364 \\
\hline
\end{tabular}

From Table 4, it can be concluded that the greater the $\mathrm{P}$ value, the larger the coverage of the droplets, which indicates that the amount of spray changed significantly under the influence of the $\mathrm{P}$ value. It proved that this variable spray system is feasible. The larger the duty cycle of the PWM signal, the smaller the variation coefficient of the droplet diameter and the better the uniformity of the droplet distribution.

\subsubsection{System overall spraying}

To verify the effectiveness of the system under adaptive and fuzzy control strategies, $\mathrm{P}$ values and actual flow values are constantly recorded during the operation. The relationship between $\mathrm{P}$ and actual flow response is shown in Figure 15. "VoTF" represented the target flow value, and "VoAF" represented the actual flow value.

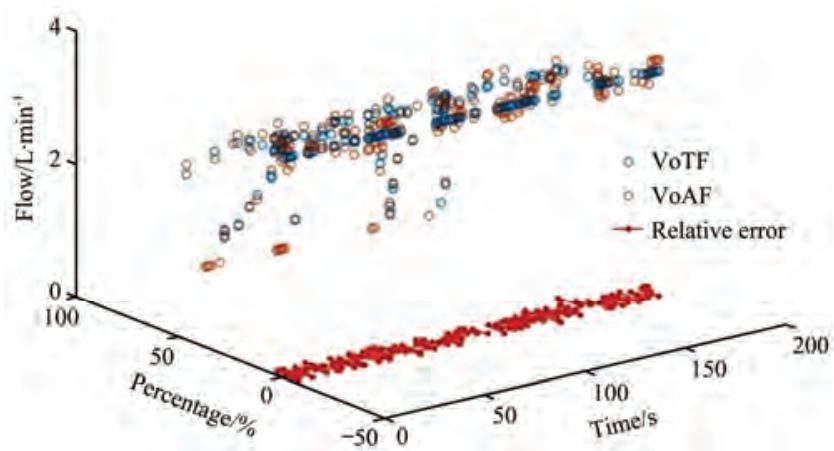

Figure 15 Fuzzy control test flow response results

In Figure 15, the VoAF of the fuzzy control system changed with the $\mathrm{P}$ value accurately. What's more, the absolute error of all VoAFs was less than $0.15 \mathrm{~L} / \mathrm{min}$ and the average relative error rate was within $2.54 \%$, which asserted the high accuracy of the variable rate spraying control system.

\section{Conclusions}

There is a common issue that UAV spraying system wastes a large amount of pesticides because of the empty areas in the crop fields. Thus, a novel vision-based spraying system which could identify empty areas automatically while spraying a precise amount of pesticides on the target regions was proposed in this study. The main findings of the experiment are summarized as below:

(1) The Lucy-Richardson algorithm sharpened the blurred image, which laid the foundation for subsequent image segmentation and feature parameter extraction. The $k$-means algorithm could effectively segment empty areas from aerial images of rice. The segmented images played a key role in feature extraction and $\mathrm{P}$ value calculation.

(2) Twenty-two feature parameters were extracted in color and texture spaces, and the SVM classifier was designed. The extracted feature parameters were input to the SVM to recognize empty areas from aerial images. Stepwise discriminant method was used to test the contribution degree in all characteristic parameters. And 17 optimal feature parameters were selected. The optimal hyperplane was trained by the 17 optimal feature parameters, which was used as the input of the SVM. The recognition rate of this hyperplane for the $375 \times 290$ pixels test samples reached $72.69 \%$ and the response time was $0.116 \mathrm{~s}$. In the acceptable loss range of detection accuracy, this algorithm could meet the real-time response requirements of the embedded system.

(3) A fuzzy controller and control rules was designed after analyzing the structure of the diaphragm pump. The established model was transplanted to an on-board embedded variable rate spraying system for spray testing. The results showed that the larger the $p$ value, the greater the droplet coverage, and the variable spray system is feasible. The larger the duty cycle of the PWM signal, the smaller the variation coefficient of the droplet diameter and the better the uniformity of the droplet distribution.

Possible work in the future is as follows: 1) to resolve high bias and enhance recognition rate by adding feature parameters and using polynomials because of the low recognition rate of the training set and test set in the article; 2) to design an intelligent target spraying gimbal which can adjust the target gimbal's direction accurately towards the empty areas in an image of which the location and size are identified and positioned by the CNN network, while controlling the flow of the pesticides to achieve more precise adaptive spraying control.

\section{Acknowledgements}

We acknowledge that the research was financially supported by Research and Demonstration of Key Technologies for Water Saving in Irrigation Areas in Guangdong Province based on UAV (2016-18), Research and Demonstration of Key Technology for Precision Spraying of Agricultural UAV based on Multi-source Information (201803020022).

\section{[References]}

[1] Rao H H, Ji C Y. Research on spray precisely toward crop-rows based on machine vision. Proceedings of First IFIPTC 12 International Conference on Computer and Computing Technologies in Agriculture (CCTA2007) (II). Boston: Springer, 2008; pp.1435-1439.

2] Zhou Z Y, Yuan W, Chen S D. Current status and future directions of rice plant protection machinery in China. Guangdong Agricultural Sciences, 2014; 15: 178-183. (in Chinese)

[3] Yang X J, Yan H R, Xu S Z. Current situation and development trend of equipment for crop protection. Transactions of the CSAM, 2002; 33(6): 130-137. (in Chinese)

[4] Xue X Y, Lan Y B. Agricultural aviation applications in USA. Transactions of the CSAM, 2013; 44(5): 194-201. (in Chinese)

[5] Lan Y B, Chen S D, Fritz B K. Current status and future trends of precision agricultural aviation technologies. Int J Agric \& Biol Eng, 2017; 10(3): 1-17.

[6] Fu Z T, Qi L J, Wang J H. Developmental tendency and strategies of precision pesticide application techniques. Transactions of the CSAM, 2007; 38(1): 189-192.

[7] Zhang Y L, Lan Y B, Bradley K. Development of aerial electrostatic spraying systems in the United States and applications in China. Transactions of the CSAE, 2016; 32(10): 1-7. 
[8] Yang F B, Xue X Y, Zhang L, Sun Z. Numerical simulation and experimental verification on downwash air flow of six-rotor agricultural unmanned aerial vehicle in hover. Int J Agric \& Biol Eng, 2017; 10(4): $41-53$.

[9] He X K, Bonds J, Herbst A, Langenakens J. Recent development of unmanned aerial vehicle for plant protection in East Asia. Int J Agric \& Biol Eng, 2017; 10(3): 18-30.

[10] Huang Y B, Hoffmann C, Fritz B, Lan Y B. Development of an unmanned aerial vehicle-based spray system for highly accurate site-specific application. ASABE Meeting Presentation, 2008; Paper number: 083909.

[11] Xu X, Xu S, Liu Y X, Chen J S, Cai Z X, Yu Z S. Variable pesticide spraying system design based on small UAV. Guangdong Agricultural Sciences, 2014; 9: 207-210. (in Chinese)

[12] Ru Y, Jin L, Jia Z C, Bao R, Qian X D. Design and experiment on electrostatic spraying system for unmanned aerial vehicle. Transactions of the CSAE, 2015; 31(8): 42-47. (in Chinese)

[13] Zhang Y L, Lian Q, Zhang W. Design and test of a six-rotor unmanned aerial vehicle (UAV) electrostatic spraying system for crop protection. Int J Agric \& Biol Eng, 2017; 10(6): 68-76.

[14] Shahemabadi A R, Moayed M J. An algorithm for pulsed activation of solenoid valves for variable rate application of agricultural chemicals. IEEE International Symposium on Information Technology, 2008; 4: 1-3.

[15] Wang D S, Zhang J X, Li W, Xiong B, Zhang S L, Zhang W Q. Design and test of dynamic variable rate spraying system of plant protection UAV. Transactions of the CSAM, 2017; 48(5): 86-93. (in Chinese)

[16] Peña J M, Torres-Sánchez J, de Castro A I, Kelly M, López-Granados F. Weed mapping in early-season maize fields using object-based analysis of unmanned aerial vehicle (UAV) images. PLoS ONE 2013; 8(10): e77151.

[17] Hung C, Xu Z, Sukkarieh S. Feature Learning Based Approach for Weed Classification Using High Resolution Aerial Images from a Digital Camera Mounted on a UAV. Remote Sensing, 2014; 6: 12037-12054.

[18] Rani R U, Amsini P. Pest identification in leaf images using SVM classifier. International Journal of Computational Intelligence and Informatics, 2016; 6(1): 248-260.

[19] Su, Y X, Xu H, Yan L J. Support vector machine-based open crop model (SBOCM): Case of rice production in China. Saudi Journal of Biological
Sciences, 2017; 24(3): 537-547.

[20] Pedram Ghamisi, Jon Atli Benediktsson, magnus orn ulfarsson. spectral-spatial classification of hyperspectral images based on hidden Markov random fields. Browse Journals \& Magazines, 2013; 52(5): 2565-2574.

[21] Medar R A, Rajpurohit V S. A survey on data mining techniques for crop yield prediction. International Journal of Advance Research in Computer Science and Management Studies, 2014; 2(9): 59-64

[22] Tian Y W, Li T L, Li C H, Piao Z L, Sun G K, Wang B. Image recognition method for grape diseases based on support vector machine. Transactions of the CSAE, 2007; 23(6): 175-180. (in Chinese)

[23] Camargo A, Smith J S. Image pattern classification for the identification of disease causing agents in plants. Computers and Electronics in Agriculture, 2009; 66(2): 121-125.

[24] Dobesa M, Machala L, Furstc T. Blurred image restoration: A fast method of finding the motion length and angle. Digital Signal Processing, 2010; 20(6): 1677-1686.

[25] Wagstaff K, Cardie C. Constrained $k$-means clustering with background knowledge. Proceedings of the Eighteenth International Conference on Machine Learning, 2001; 1: 577-584.

[26] Han D, Wu P, Zhang Q, Han G D, Tong F. Feature extraction and image recognition of typical grassland forage based on color moment. Transactions of the CSAE, 2016; 32(23): 168-175

[27] Yang K Z, Cheng Y L. A method of SAR image texture feature extraction based on co-occurrence matrix. Electronic Technology, 2011; 24(10): 66-69.

[28] He Q. Research on support vector machines in embedded image recognition. Hangzhou: Hangzhou Dianzi University, 2015.

[29] Yan G Y, Ma X D. Research on the calculation method of plant leaf area based on images. Journal of Jiamusi University, 2009; 27(2): 201-203.

[30] Guo N, Hu J T. Design and experiment of variable rate spaying system on smith-fuzzy PID control. Transactions of the CSAE, 2014; 30(8): 56-64.

[31] Wan J F, Li D, Tu Y Q, Zhang C H. Performance analysis model for real-time Ethernet-based computer numerical control system. Journal of Central South University of Technology, 2011; 18(5): 1545-1553.

[32] Ma Y J, Zhang Y, Wan J F, Zhang D Q, Pan N. Robot and cloud-assisted multi-modal healthcare system. Cluster Computing, 2015; 18(3): 1295-1306. 